\title{
The oncogenic role of NKAP in the growth and invasion of colon cancer cells
}

\author{
WEIBIN SHU ${ }^{1}$, GUOQIN LIU ${ }^{2}$, YUNPENG DAI ${ }^{3}$, ALEI FENG $^{1}$, ZHENG CHEN $^{1}$, JUNQING HAN ${ }^{1}$ and XINYU LI ${ }^{3}$ \\ ${ }^{1}$ Department of Oncology, Shandong Provincial Hospital Affiliated to Shandong University; \\ ${ }^{2}$ Department of General Surgery, Jinan Central Hospital Affiliated to Shandong University; \\ ${ }^{3}$ Department of Pediatrics, Shandong Provincial Hospital Affiliated to Shandong University, \\ Jinan, Shandong 250021, P.R. China
}

Received January 21, 2019; Accepted July 16, 2019

DOI: $10.3892 /$ or.2019.7322

\begin{abstract}
It has been reported that $\mathrm{NF}-\kappa \mathrm{B}$ activating protein (NKAP) is a transcriptional repressor of the Notch signaling pathway and is involved in the proliferation and survival of hematopoietic stem cells. In the present study, we aimed to investigate the effect of NKAP on the progression and metastasis of colon cancer. The results of immunohistochemical staining and western blot analysis showed that NKAP was upregulated in colon cancer tissues, and its expression was associated with colon cancer stages. CCK-8, colony formation, Transwell, and flow cytometry assays were used to demonstrate that NKAP knockdown significantly suppressed the proliferation and invasion of HCT116 and HT-29 cells, and also induced apoptosis and autophagy. By contrast, NKAP overexpression markedly promoted the proliferation and invasion of HCT-15 cells, and inhibited cell apoptosis and autophagy. Moreover, we observed that NKAP knockdown inhibited the epithelial-mesenchymal transition (EMT) process in HCT116 and HT-29 cells, while NKAP overexpression promoted EMT in HCT-15 cells. Furthermore, NKAP knockdown inhibited activation of the Akt/mTOR signaling pathway by downregulating the phosphorylation of Akt and mTOR, as well as their downstream proteins, whereas NKAP overexpression promoted the Akt/mTOR signaling pathway. Additionally, expression of P65 was downregulated by silencing of NKAP and upregulated by NKAP overexpression. These data suggest
\end{abstract}

Correspondence to: Dr Junqing Han, Department of Oncology, Shandong Provincial Hospital Affiliated to Shandong University, 324 Jingwu Road, Jinan, Shandong 250021, P.R. China

E-mail: hanjq19601960@163.com

Dr Xinyu Li, Department of Pediatircs, Shandong Provincial Hospital Affiliated to Shandong University, 324 Jingwu Road, Jinan, Shandong 250021, P.R. China

E-mail: lixinyu0627@163.com

Key words: colon cancer, NKAP, progression, metastasis, Akt/mTOR pathway that NKAP functions as an oncogene in the growth and invasion of colon cancer in vitro.

\section{Introduction}

Colon cancer is ranked as the third most common cancer among men and the second most common cancer among women in the world, with the highest incidence between 40 and 50 years of age (1). Among adults less than 50 years of age, the incidence of colon cancer has increased by $22 \%$ from 2000 to 2013 , and the rate of mortality has increased by $13 \%$ from 2000 to 2014 (2). Metastasis is the leading cause of death in colon cancer patients, and $\sim 50 \%$ of these patients develop metastatic disease $(3,4)$. Therefore, it is necessary to understand the mechanism of cancer metastasis in order to identify new targets to suppress cancer metastasis.

$\mathrm{NF}-\kappa \mathrm{B}$ activating protein (NKAP) is a conserved protein and is named based on its ability to enhance NF- $\kappa \mathrm{B}$ activity (5). Previous research has revealed that NKAP is a transcriptional repressor of the Notch signaling pathway and plays an essential role in the development of T cells and invariant NKT (iNKT) cells, as well as $\mathrm{T}$ cell maturation and the acquisition of functional competency (6-8). NKAP is also crucial for maintenance and survival of hematopoietic stem cells (HSCs), and its deficiency could result in decreased proliferation and increased apoptosis in HSCs (9). Thapa et al found that NKAP is also required for proliferation burst and differentiation of NKT17 cells (10). Additionally, a new role for NKAP as a nuclear speckle protein in RNA splicing and processing in HeLa cells has been reported (11). NKAP is also reported to play a role in the regulation of mitosis, and its dysregulation can lead to chromosomal instability, which may result in tumorigenesis (12).

In this study, we aimed to determine whether NKAP was upregulated in colon cancer. To further investigate whether NKAP was involved in the progression and metastasis of colon cancer, we knocked down the expression of NKAP in HCT116 and HT-29 cells and upregulated its expression in HCT-15 cells. Our data showed that NKAP knockdown resulted in a significant decrease in the proliferation and invasion of colon cancer cells, as well as induction of apoptosis and autophagy, whereas NKAP overexpression promoted cell proliferation and invasion, and inhibited apoptosis and autophagy. 


\section{Materials and methods}

Tissue specimens. The Research Ethics Committee of Shandong Provincial Hospital Affiliated to Shandong University (no. 2018-220) approved the present study. A total of 75 cases of colon cancer tissues and corresponding paracancerous tissues were obtained from patients who underwent colon cancer resection at Shandong Provincial Hospital from June 1, 2013 to September 30, 2016 for immunohistochemical staining. The patient ages ranged from 26-83 years (median, 63.5 years), and included 43 males and 32 females. The clinicopathological features of colon cancer patients are shown in Table I. An additional 25 pairs of colon cancer tissues and corresponding paracancerous tissues were collected for western blot assay. Written informed consent was obtained from all the patients. All the specimens remained anonymous throughout the study and were handled according to the ethical and legal standards of the hospital.

Cell culture and infection. Human colon cancer cell lines HCT116, LoVo, SW480 and HCT-15 and colorectal adenocarcinoma cell line HT-29 (STR profiling was used for authentication), were obtained from the Cell Bank of the Chinese Academy of Sciences (Shanghai, China). Cells were cultured in RPMI-1640 (HyClone, Thermo Fisher Scientific) medium containing 10\% FBS (Gibco, Thermo Fisher Scientific) at $37^{\circ} \mathrm{C}$ with $5 \% \mathrm{CO}_{2}$. The sequence of shRNA targeting NKAP was obtained from Ribobio (Guangzhou). Virapower system (Invitrogen, Thermo Fisher Scientific) was used for lentivirus packaging to construct stably expressing shRNA-NKAP or shRNA control (negative control, NC) (12). NKAP cDNA sequences were cloned in pcDNA3.1 plasmid then transfected into HCT-15 cells, and the empty plasmid was used as negative control. Cells at 40-50\% confluency were infected with lentivirus expressing shRNA-NKAP or pcDNA3.1-NKAP, and shRNA control or pcDNA3.1 was used as the negative control. Infected cells were harvested for drug selection with puromycin, and cells stably expressing shRNA or NKAP were used for subsequent experiments.

Immunohistochemical staining. Tissues were stained using the EliVision ${ }^{\mathrm{TM}}$ plus kit (Maixin, Fuzhou, China) according to the manufacturer's instructions (13). Anti-NKAP was obtained from Abcam. The percentage of positive stained cells was graded as follows: $1,6-25 \% ; 2,26-50 \% ; 3,51-75 \% ; 4,>75 \%$ and $0, \leq 5 \%$. The staining intensity was graded as follows: No staining (0), weak staining (faintly yellow; 1 ), moderate staining (brownish yellow; 2), and strong staining (brown; 3). The percentage of NKAP-positive cells multiplied with the staining intensity was the final score of samples. Colon cancer patients were divided into two groups based on this score, in which scores $\leq 6$ were regarded as NKAP low-expression group, and scores $>6$ were regarded as NKAP high-expression group.

Reverse transcription-PCR (RT-PCR). Total RNA was extracted using TRIzol (PuFei) and reverse transcribed to cDNA using SuperRT cDNA Synthesis kit (CWBIO). The reaction system was as follows: dNTP Mix, $4 \mu \mathrm{l}$; primer Mix,
$2 \mu \mathrm{l}$; mRNA, $2 \mu \mathrm{l}$; RT buffer, $4 \mu \mathrm{l}$; SuperRT, $1 \mu \mathrm{l}$; RNase-Free water, $7 \mu \mathrm{l} ; 42^{\circ} \mathrm{C}$ for $30 \mathrm{~min} ; 85^{\circ} \mathrm{C}$ for $5 \mathrm{~min}$. The NKAP mRNA expression was detected using SYBR Master Mixture (Takara Bio Inc.). The reaction conditions were as follows: $95^{\circ} \mathrm{C}$ for $30 \mathrm{sec}$; and $95^{\circ} \mathrm{C}$ for $5 \mathrm{sec}, 55^{\circ} \mathrm{C}$ for $30 \mathrm{sec}$ and $72^{\circ} \mathrm{C}$ for $30 \mathrm{sec}$ for 40 cycles. Primers used were: NKAP: upstream, $5^{\prime}-\mathrm{CGG}$ CAGAAGAGATTAAGTGAG-3', downstream, 5'-CTCCAC TGGTGTATGTTCATC-3'; GAPDH: upstream, 5'-TGACTT CAACAGCGACACCCA-3', downstream, 5'-CACCCTGTT GCTGTAGCCAAA-3'. The comparative $\mathrm{Cq}(\Delta \Delta \mathrm{cq})$ method was used to analyze the obtained data (14).

Western blot assay. RIPA lysis buffer (CWBIO) was used for cell lysis in order to perform protein extraction, and protein concentration was measured with a bicinchoninic acid protein assay kit (Beyotime, Beijing, China). Protein $(20 \mu \mathrm{g})$ was separated by $10 \%$ SDS-PAGE gel and then transferred to polyvinylidene fluoride membranes (PVDF). The primary antibodies were added for incubation with the membrane in 5\% non-fat milk in TBST ( $150 \mathrm{mM} \mathrm{NaCl}, 20 \mathrm{mM}$ Tris, $0.05 \%$ Tween-20) at $4^{\circ} \mathrm{C}$ overnight. Hereafter, the membrane was incubated with secondary antibodies for $1 \mathrm{~h}$ and treated with an enhanced chemiluminescence kit for signal development. ImageJ software was performed to quantify the density of each band. The primary antibodies, which were obtained from Abcam, were as follows: Anti-NKAP (rabbit polyclonal; dilution 1:1,000; cat. no. ab229096), anti-Bcl-2 (rabbit polyclonal; dilution 1:1,000; cat. no. ab59348), anti-Bax (rabbit polyclonal; dilution 1:1,000; cat. no. ab53154), anti-p-Akt (rabbit monoclonal; dilution 1:1,000; cat. no. ab81283), anti-Akt (rabbit monoclonal; dilution 1:1,000; cat. no. ab32505), anti-mTOR (rabbit polyclonal; dilution 1:1,000; cat. no. ab2732), anti-p-mTOR (rabbit polyclonal; dilution 1:1,000; cat. no. ab131538), anti-P70 (Rabbit monoclonal; dilution 1:1,000; cat. no. ab32529), anti-Cyclin D1 (rabbit monoclonal; dilution 1:1,000; cat. no. ab134175), anti- $\beta$-catenin (rabbit monoclonal; dilution 1:1,000; cat. no. ab32572), anti-E-cad (rabbit polyclonal; dilution 1:1,000; cat. no. ab15148), anti-P62 (rabbit polyclonal; dilution 1:1,000; cat. no. ab91526), anti-Beclin1 (rabbit polyclonal; dilution 1:1,000; cat. no. ab62557), anti-LC3B (rabbit polyclonal; dilution 1:1,000; cat. no. ab48394), and anti-GAPDH (rabbit polyclonal; dilution 1:1,000; cat. no. ab9485). The anti-active Caspase-3 p17-specific (rabbit polyclonal; dilution 1:1,000; cat. no. 19677-1-AP) and all secondary antibodies (anti-rabbit IgG; dilution 1:2,000; cat. no. SA00001-2) were obtained from Proteintech Group (Rosemont).

CCK-8 assay. After infection, the cells were seeded in 96-well plates at a density of $1 \times 10^{3} /$ well and cultured for $72 \mathrm{~h}$. Then, $10 \mu 1$ of CCK-8 reagent (Solarbio Science \& Technology) was added into each well and incubated for $90 \mathrm{~min}$ at $37^{\circ} \mathrm{C}$. The OD value of excitation light was detected using enzyme standard instrument at a wavelength of $450 \mathrm{~nm}$.

Colony formation assay. After infection for $24 \mathrm{~h}, 5 \times 10^{2}$ cells were seeded into a $10 \mathrm{~cm}$ dish containing $5 \mathrm{ml}$ of medium and cultured at $37^{\circ} \mathrm{C}$ with $5 \% \mathrm{CO}_{2}$ until cells had formed sufficiently large clones. The medium was removed, and colonies were stained with $0.1 \%$ crystal violet for $30 \mathrm{~min}$. Images were captured and the number of clones was counted. 
Table I. Associations between the NKAP expression levels and the clinicopathological characteristics of colon cancer patients.

\begin{tabular}{|c|c|c|c|c|}
\hline \multirow[b]{2}{*}{$\begin{array}{l}\text { Clinicopathological } \\
\text { characteristics }\end{array}$} & \multirow[b]{2}{*}{$\begin{array}{l}\text { Cases } \\
\mathrm{n}=75\end{array}$} & \multicolumn{2}{|c|}{$\begin{array}{l}\text { NKAP } \\
\text { expression }\end{array}$} & \multirow[b]{2}{*}{ P-value } \\
\hline & & $\begin{array}{l}\text { High } \\
(n=41)\end{array}$ & $\begin{array}{c}\text { Low } \\
(\mathrm{n}=34)\end{array}$ & \\
\hline \multicolumn{5}{|l|}{ Age (years) } \\
\hline$\leq 60$ & 26 & 12 & 14 & 0.2807 \\
\hline$>60$ & 49 & 29 & 20 & \\
\hline \multicolumn{5}{|l|}{ Sex } \\
\hline Male & 43 & 27 & 16 & 0.1013 \\
\hline Female & 32 & 14 & 18 & \\
\hline \multicolumn{5}{|l|}{ Tumor size (cm) } \\
\hline$\leq 3$ & 14 & 4 & 10 & 0.0296 \\
\hline$>3$ & 61 & 37 & 24 & \\
\hline \multicolumn{5}{|l|}{ Distant metastasis } \\
\hline Yes & 9 & 5 & 4 & 0.9545 \\
\hline No & 66 & 36 & 30 & \\
\hline \multicolumn{5}{|c|}{ Lymph node metastasis } \\
\hline Yes & 36 & 20 & 16 & 0.8819 \\
\hline No & 39 & 21 & 18 & \\
\hline \multicolumn{5}{|l|}{ Stage } \\
\hline $\mathrm{I}+\mathrm{II}$ & 13 & 3 & 10 & 0.0119 \\
\hline III+IV & 62 & 38 & 24 & \\
\hline
\end{tabular}

Invasion assay. Cell invasion experiments were performed using Transwell chambers coated with Matrigel. Infected cells were cultured in serum-free medium for $24 \mathrm{~h}$ and digested. Cells $\left(1 \times 10^{4}\right)$ were transferred to the top chamber, and the complete medium was added into the lower chamber. After $24 \mathrm{~h}$ of incubation, the invasive cells were fixed with $4 \%$ paraformaldehyde at room temperature for $30 \mathrm{~min}$. The filters were then stained with $0.1 \%$ crystal violet at room temperature for another $20 \mathrm{~min}$. The invasive cells were captured and counted under the dissecting microscope (magnification, x100).

Apoptosis detection assay. Apoptosis of infected cells was determined using Annexin V PE/7-AAD kit (KeyGen BioTECH). Infected cells were harvested and resuspended in $1 \mathrm{X}$ binding buffer at a density of $1-5 \times 10^{6}$ cells $/ \mathrm{ml}$. Next, $100 \mu \mathrm{l}$ cell suspensions were incubated with Annexin V PE/7-AAD apoptosis kit for staining. The samples were analyzed using the FACS caliber instrument. The rate of apoptosis was analyzed by BD FACSDiva software.

Statistical analysis. Analyses were performed using SPSS 18.0 software. Data were obtained from three independent experiments and expressed as mean \pm standard deviation. The Chi-square test was used to evaluate associations. Comparisons between the two groups were analyzed using Student's t-test. $\mathrm{P}<0.05$ was considered statistically significant.

\section{Results}

NKAP is upregulated in colon cancer and its expression is associated with clinicopathological parameters of colon cancer. Immunohistochemistry assay was performed to investigate the expression of NKAP in colon cancer. As shown in Fig. 1A, the NKAP-positive signal was located in the nucleus and cytoplasm of tumor cells. NKAP expression in colon cancer tissues $(54.67 \%, 41 / 75)$ was much higher than NKAP expression in paracancerous tissues $(17.33 \%, 13 / 75)$. Moreover, the expression of NKAP was positively associated with tumor size and stages (both $\mathrm{P}<0.05$, Table I). Western blot analysis revealed that the protein expression of NKAP was significantly upregulated in colon cancer tissues compared to paracancerous tissues $(\mathrm{P}<0.05$, Fig. $1 \mathrm{~B}, \mathrm{n}=25)$.

NKAP regulates the proliferation and viability of colon cancer cells. We observed high expression of NKAP mRNA in HT-29 and HCT116 cells, and low expression of NKAP in HCT-15 cells Fig. S1A). In order to investigate the role of NKAP in the progression of colon cancer, NKAP expression was knocked down by shRNA interference in HCT116 and HT-29 cells at the mRNA (Fig. 2A) and protein (Fig. 2B) levels. HCT-15 cells were transfected with pcDNA3.1-NKAP plasmid to upregulate its expression (Fig. 2A and B). Furthermore, CCK-8 assay was performed to examine the effect of NKAP on proliferation in colon cancer cells. Our data showed that after $72 \mathrm{~h}$ of incubation, the proliferation of HCT116 cells with shNKAP interference was clearly inhibited compared to the NC ( $<<0.05$, Fig. 2C). Similarly, NKAP knockdown also reduced the proliferation of colorectal adenocarcinoma HT-29 cells $(\mathrm{P}<0.05$, Fig. 2D). As shown in Fig. 2E, upregulation of NKAP significantly increased the proliferation of HCT-15 cells $(\mathrm{P}<0.05)$. The colony formation assay demonstrated a significant decrease in colony forming ability. NKAP knocked down HCT116 and HT-29 cells compared to the NC, while an increase in colony forming ability was observed in HCT-15 cells with NKAP overexpression (both $\mathrm{P}<0.05$, Fig. $2 \mathrm{~F}$ and $\mathrm{G}$ ). Taken together, these results indicated that NKAP played an oncogenic role in the proliferation and viability of colon cancer cells in vitro.

NKAP regulated the invasion and epithelial-mesenchymal transition (EMT) of colon cancer cells. Metastatic potential is one of the most important features of all tumors. Therefore, we examined the effect of NKAP on colon cancer invasion using Transwell assay. As shown in Fig. 3A and C, silencing of NKAP significantly reduced the invasion of HCT116 and HT-29 cells (both $\mathrm{P}<0.05$ ) compared to NC cells. Consistently, NKAP overexpression markedly resulted in an increase in invasion in HCT-15 cells (Fig. 3B and C). Furthermore, we observed significant upregulation of adhesion factor $\beta$-catenin in colon cancer tissues (Fig. S1C and D), and it was significantly downregulated in NKAP-deficient cells but upregulated in NKAP overexpression cells (Fig. 3D). The epithelial marker protein E-cad was upregulated in NKAP-silenced cells, while it was markedly downregulated in NKAP overexpressed HCT-15 cells (Fig. 3D). This was an indication that NKAP regulated the EMT process in colon cancer cells in vitro. 

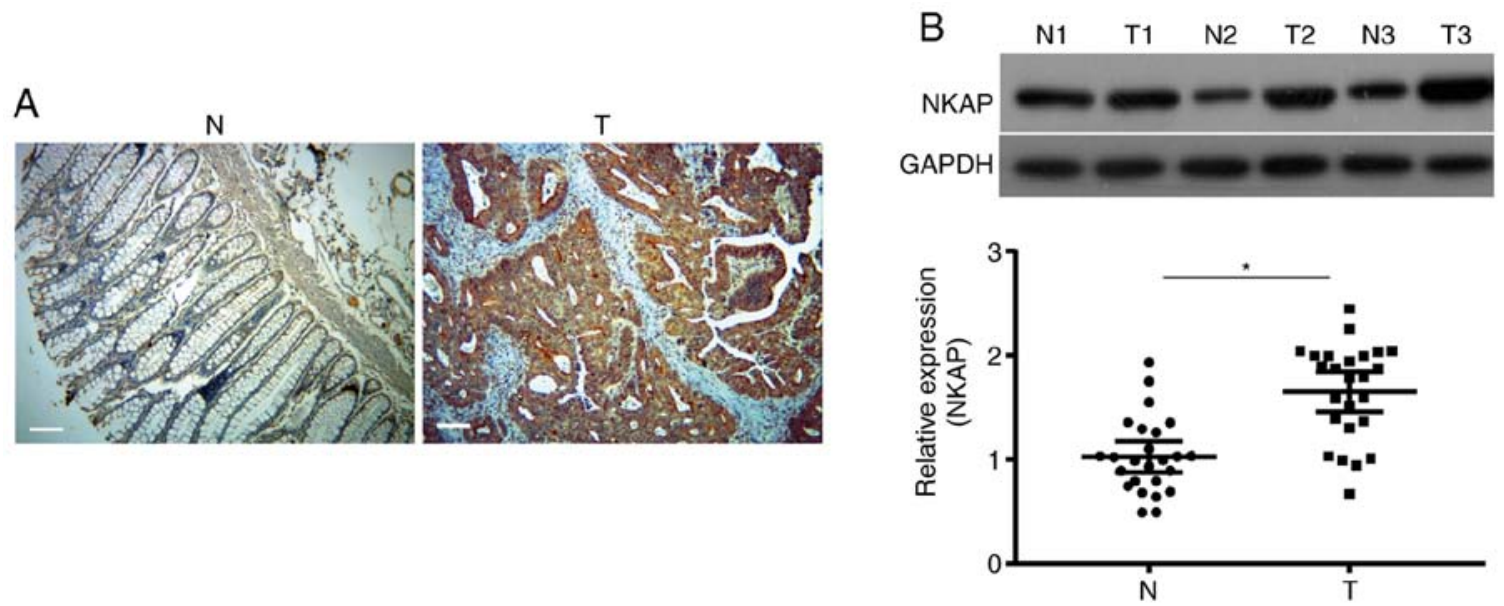

Figure 1. NKAP is upregulated in colon cancer. (A) Expression of NKAP in colon cancer tissue (T) and paracancerous tissue (N) using immunohistochemistry assay: x100 magnification, scale bar: $100 \mu \mathrm{m}$. (B) Expression of NKAP in colon cancer tissue (T1-T3) and paracancerous tissue (N1-N3) using western blot analysis. N: paracancerous tissue; $\mathrm{T}$ : colon cancer tissue. ${ }^{*} \mathrm{P}<0.05$ compared to paracancerous tissue.


Figure 2. NKAP regulates the proliferation and viability of HCT116, HT-29 and HCT-15 cells. HCT116 and HT-29 cells were transfected with shRNA-NKAP (sh-NKAP), shRNA control was used as negative control (sh-NC); HCT-15 cells were transfected with pcDNA3.1-NKAP (NKAP overexpression, NKAP-OE), and the empty plasmid was used as negative control (NC-OE). (A) After $24 \mathrm{~h}$ of transfection, the expression of NKAP mRNA was detected using RT-PCR in HCT116, HT-29 and HCT-15 cells. (B) Following transfection for 48 h, the expression of NKAP protein in HCT116, HT-29 and HCT-15 cells was examined using western blot analysis. (C-E) Following transfection for $72 \mathrm{~h}$, cell viability was assessed using CCK-8 assay in HCT116 (C), HT-29 (D) and HCT-15 (E) cells. (F) Cell proliferation ability was detected using colony formation assay in HCT116 (left), HT-29 (middle) and HCT-15 (right) cells. Scale bar: $1 \mathrm{~cm}$. ${ }^{*} \mathrm{P}<0.05,{ }^{* * *} \mathrm{P}<0.01$ compared with negative control (NC).

NKAP regulates apoptosis and autophagy of colon cancer cells. One hallmark of tumors is uncontrolled apoptosis (15).
Therefore, flow cytometric assay was performed in order to examine the effect of NKAP on cell apoptosis. As shown 
A

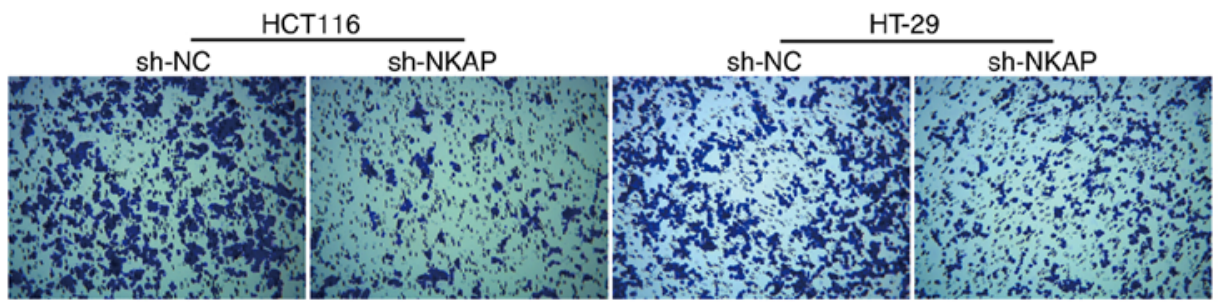

B

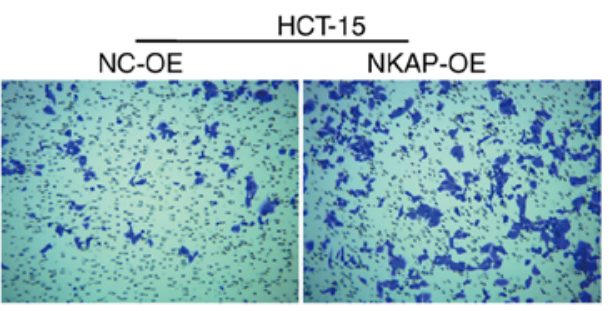

C
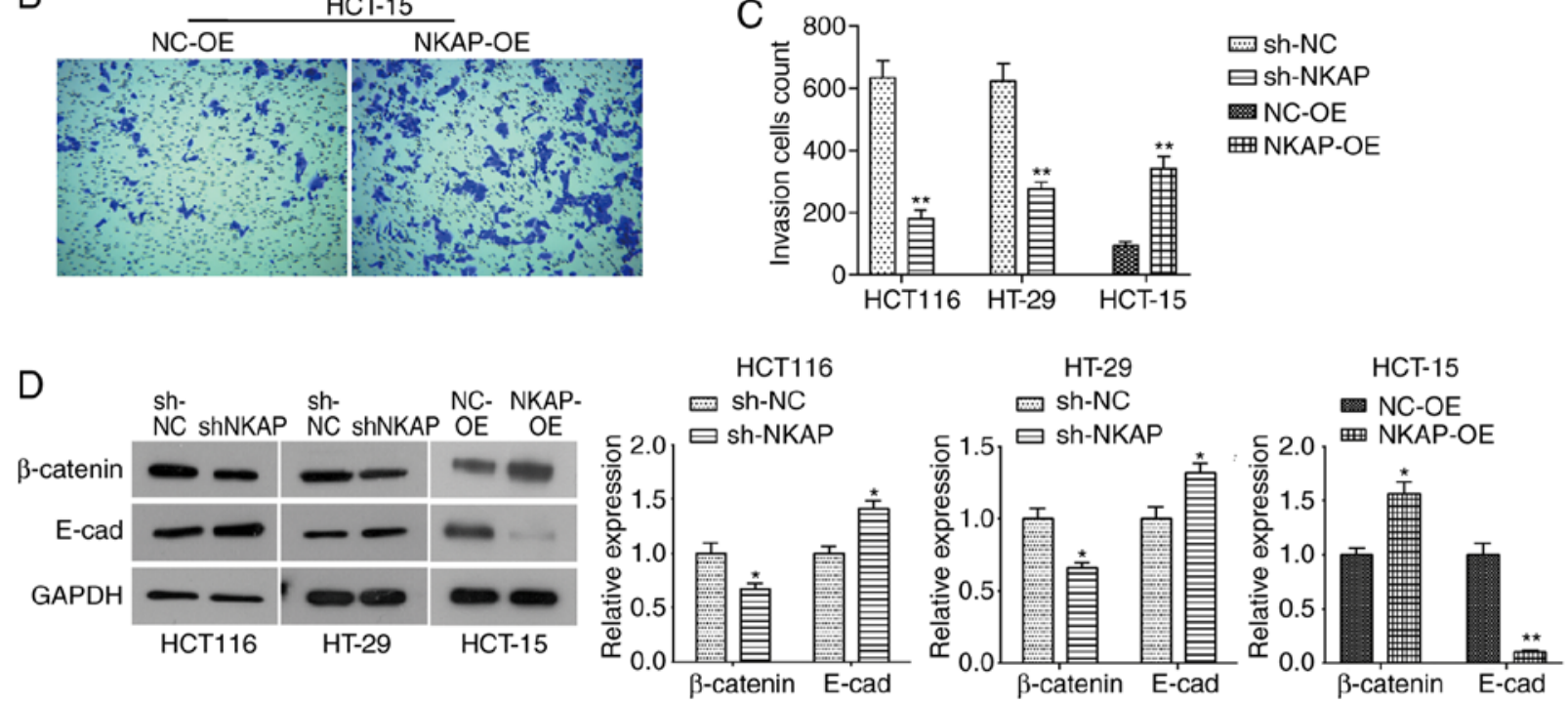

Figure 3. NKAP regulates cell invasion and EMT in colon cancer cells in vitro. (A) After $24 \mathrm{~h}$ of transfection, cell invasion abilities of HCT116 (A) and HT-29 (B) cells were assessed using Transwell assay. (B) Effect of NKAP overexpression on cell invasion in HCT-15 cells was examined using Transwell assay. (C) Quantitative analysis of Transwell results. (D) Effect of NKAP knockdown and overexpression on expression of EMT-related proteins, $\beta$-catenin and E-cad, was examined using western blot analysis. sh-NKAP, cells transfected with shRNA-NKAP; sh-NC, cells transfected with shRNA control; NKAP-OE, NKAP overexpression, cells transfected with pcDNA3.1-NKAP; NC-OE, cells transfected with the empty plasmid. ${ }^{*} \mathrm{P}<0.05,{ }^{* *} \mathrm{P}<0.01$ compared with negative control (NC).

in Fig. 4A and C, the rate of apoptosis was significantly elevated following downregulation of NKAP compared to the NC cells, both in HCT116 and HT-29 cells (both P<0.05). Consistently, NKAP overexpression inhibited apoptosis in HCT-15 cells ( $\mathrm{P}<0.05$, Fig. 4B and $\mathrm{C})$. In order to determine the related mechanism of apoptosis induction by NKAP, changes in expression of apoptosis-related proteins were assessed. Western blot results showed a significant decrease in the expression of anti-apoptotic protein Bcl-2 in NKAP-silenced cells, and a notable increase in the expression of pro-apoptotic proteins Bax and Active Caspase-3 was observed in both HCT116 and HT-29 cells interfered with shRNA-NKAP (both $\mathrm{P}<0.05$, Fig. 4D). An increase in the expression of Bcl-2 was observed in NKAP overexpression cells, as well as a decrease in the expression of Bax and Active Caspase-3 (Fig. 4D). In addition, we determined that the expression of autophagy-related proteins was modulated by NKAP. As indicated by western blotting, there was an increase in Beclin1 and LC3-II expression and a decrease in LC3-I expression in NKAP silenced cells, while a decrease in Beclin1 and LC3-II expression and increase in P62 and LC3-I expression (both $\mathrm{P}<0.05$, Fig. 4E). These data suggested that NKAP inhibited apoptosis and autophagy in colon cancer cells, while silencing its expression can promote apoptosis and autophagy.

$N K A P$ regulates activation of the Akt/mTOR and $N F-\kappa B$ signaling pathways in colon cancer cells. Considering the pivotal role of the Akt/mTOR signaling pathway in cell growth and survival, as well as tumor progression and metastasis, the expression of its key components was examined in NKAP-silenced cells. As is shown in Fig. S1B and D, the Akt/mTOR pathway was overactivated in colon cancer tissues as a result of the upregulation of p-Akt and p-mTOR expression. Our data showed that NKAP knockdown did not affect the total amount of Akt and mTOR, but it significantly reduced Akt and mTOR phosphorylation in HCT116 and HT-29 cells (Fig. 5). Accordingly, the expression of its downstream proteins, Cyclin D1 and P70, was decreased in NKAP-deficient cells compared to NC cells (Fig. 5). By contrast, upregulation of NKAP promoted Akt and mTOR phosphorylation and expression of Cyclin D1 and P70 in HCT-15 cells (Fig. 5).

Since NKAP could enhance NF- $\kappa B$ activity, we examined the effect of NKAP expression on the NF- $\mathrm{KB}$ signaling pathway in colon cancer cells. P65 is a subunit of NF- $\mathrm{KB}$, which is a crucial nuclear transcription factor involved in cellular functions. The level of P65 was increased in colon cancer tissues (Fig. S1D) and decreased in NKAP-silenced cells, while P65 expression was significantly upregulated by NKAP overexpression (Fig. 5). This indicated that the NF- $\mathrm{KB}$ signaling pathway was suppressed by NKAP knockdown and promoted by NKAP overexpression in colon cancer cells. Taken together, we can conclude that the Akt/mTOR and $\mathrm{NF}-\mathrm{KB}$ signaling pathways may be involved in the oncogenic role of NKAP in the progression of colon cancer. 
A

HCT116
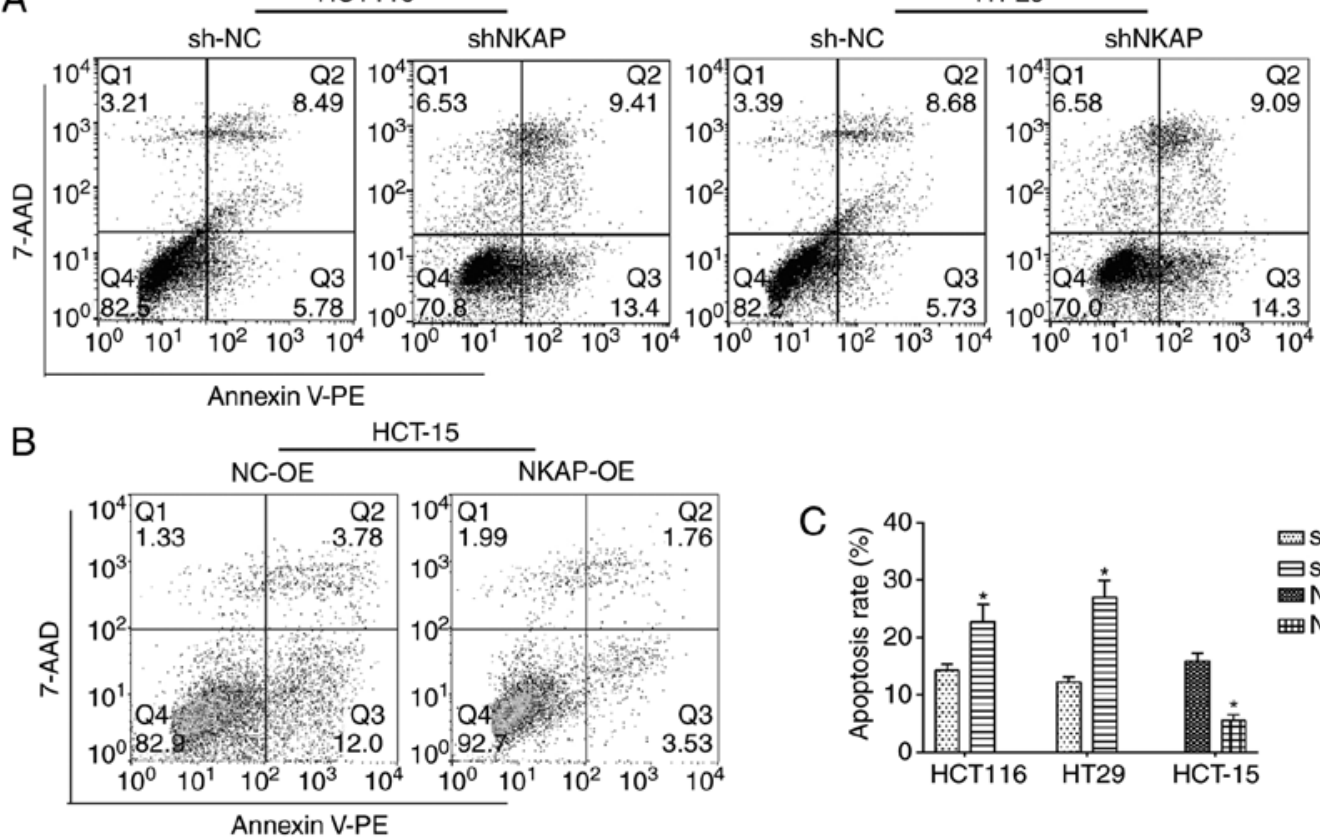

四 Sh-NC

曰 $\mathrm{ShNKAP}$

표리 NC-OE

D Sh- Sh- NC- NKAP-
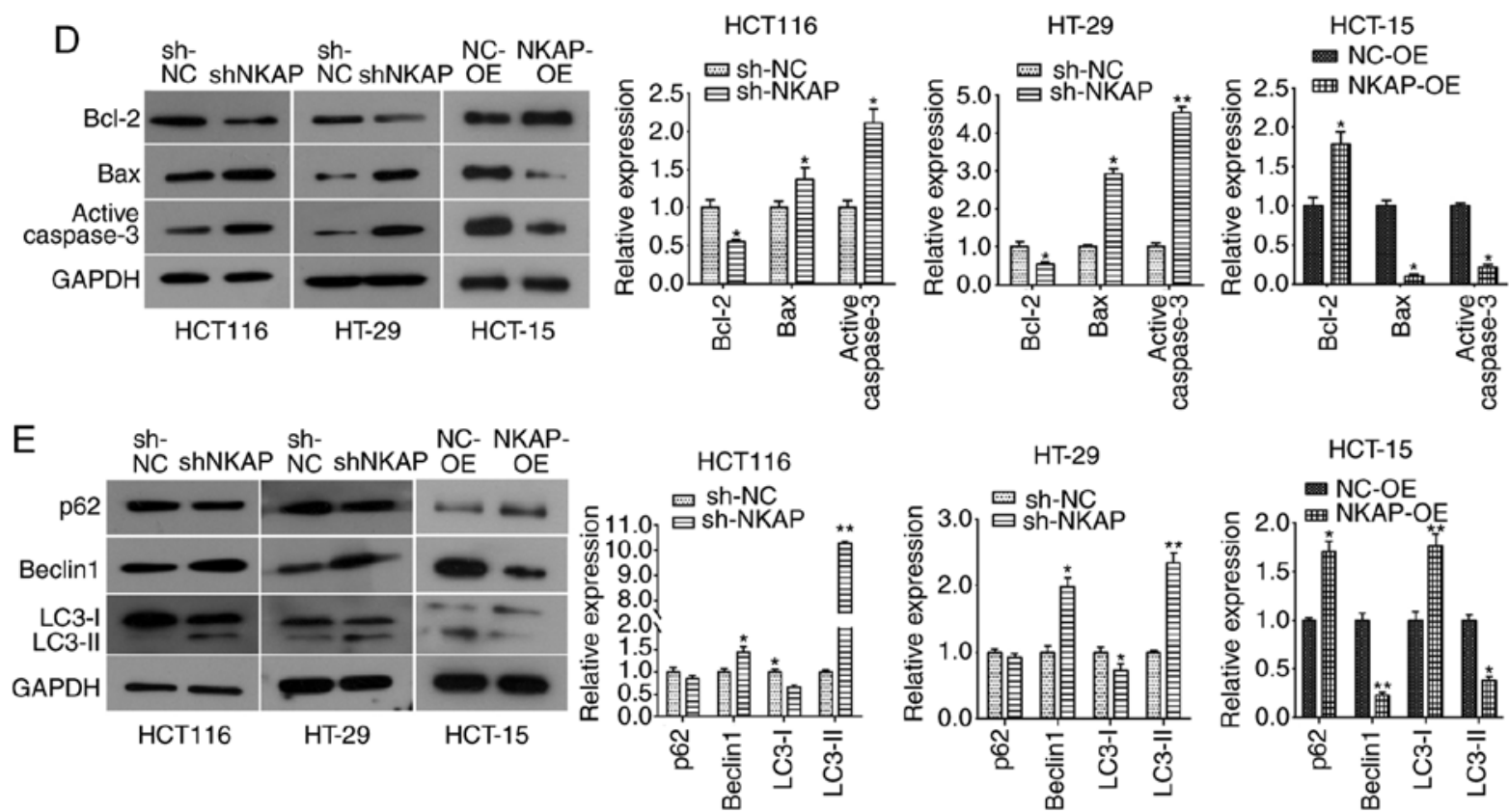

Figure 4. NKAP modulates apoptosis and autophagy in colon cancer cells. (A) Cell apoptosis of HCT116 and HT-29 cells was detected by flow cytometry. (B) Effect of NKAP overexpression on apoptosis in HCT-15 cells. (C) Quantitative analysis of apoptosis results. (D) Western blot analysis was performed to detect the expression of apoptosis-related proteins, Bcl-2, Bax and Active Caspase-3, in HCT116, HT-29 and HCT-15 cells. (E) Expression of autophagy-related proteins was assessed using western blotting. sh-NKAP, cells transfected with shRNA-NKAP; sh-NC, cells transfected with shRNA control; NKAP-OE, NKAP overexpression, cells transfected with pcDNA3.1-NKAP; NC-OE, cells transfected with the empty plasmid. "P<0.05, ${ }^{* *} \mathrm{P}<0.01$ compared with negative control (NC).

\section{Discussion}

It has been reported that NKAP is involved in cell development and proliferation of $\mathrm{T}$ cell and iNKT cells (6-8). A recent study has revealed that the dysregulation of NKAP results in chromosomal instability which is associated with tumorigenesis (12). However, there is still a knowledge gap regarding the role of NKAP in colon cancer progression. Herein, we observed that the expression of NKAP was upregulated in colon cancer tissues. Its expression demonstrated a positive association with tumor size and stages (Table I), suggesting potential involvement of NKAP in the progression of colon cancer. Interestingly, our data showed that NKAP knockdown significantly reduced cell proliferation and viability in HCT116 and HT-29 cells, while NKAP overexpression promoted the proliferation and viability of HCT-15 cells. As is commonly known, invasion is a common hallmark of malignant tumors, resulting in tumor metastasis, which is the leading cause of death in colon cancer patients (16). The present study showed that the invasion of colon cancer cells was inhibited by NKAP knockdown and promoted by NKAP overexpression in vitro, which was consistent with the effect of NKAP on breast cancer 


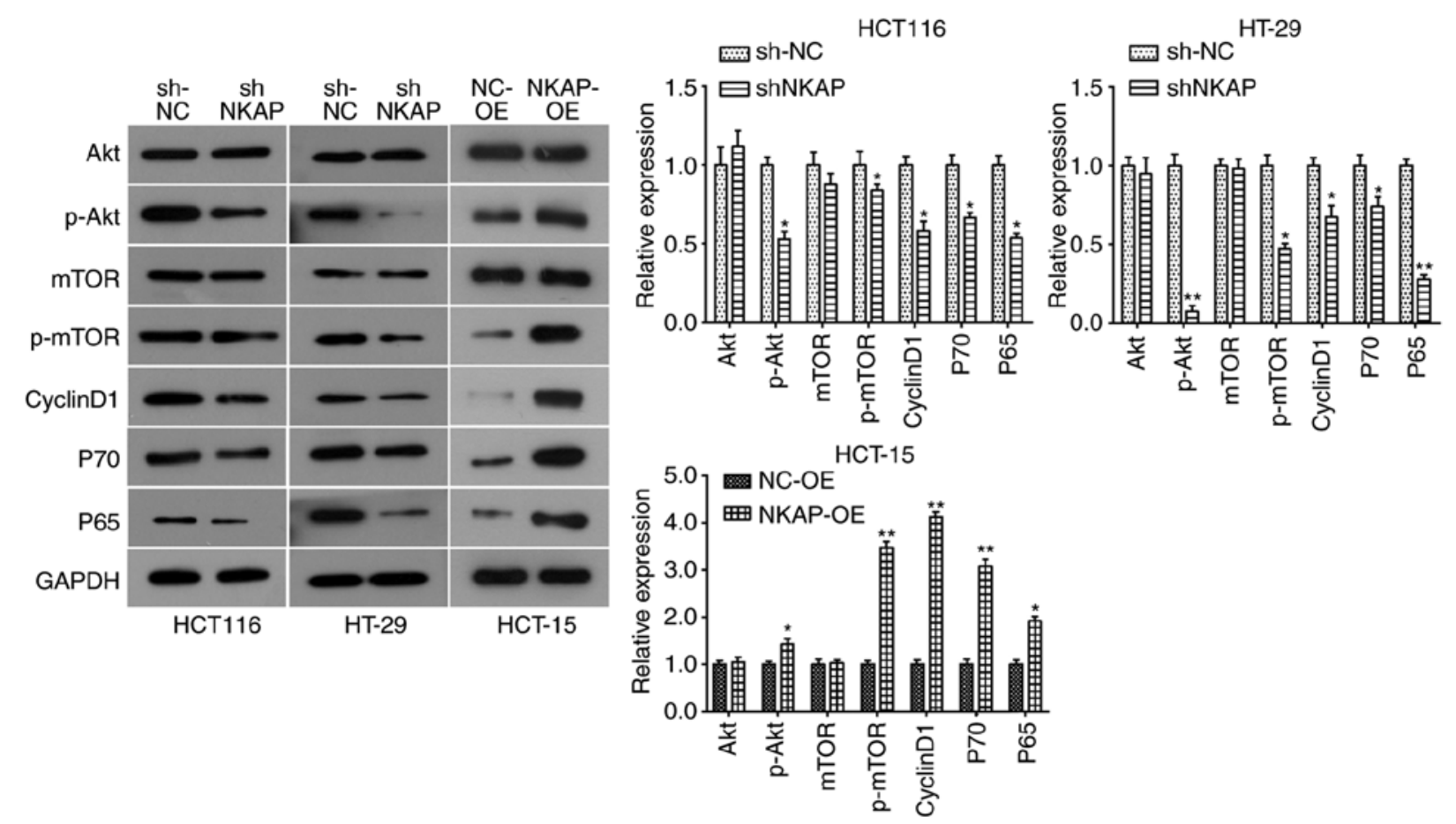

Figure 5. NKAP regulates the activation of the Akt/mTOR and NF- $\mathrm{kB}$ signaling pathways in colon cancer cells. Following transfection for $48 \mathrm{~h}$, the expression of Akt/mTOR pathway-related proteins (Akt, p-Akt, mTOR, p-mTOR, Cyclin D1, and P70) was detected using western blotting in HCT116, HT-29 and HCT-15 cells. Expression of the key component of NF- $\mathrm{B}$ s signaling pathway P65 was also assessed using western blotting. sh-NKAP, cells transfected with shRNA-NKAP; sh-NC, cells transfected with shRNA control; NKAP-OE, NKAP overexpression, cells transfected with pcDNA3.1-NKAP; NC-OE, cells transfected with the empty plasmid. ${ }^{*} \mathrm{P}<0.05,{ }^{* *} \mathrm{P}<0.01$ compared with negative control (NC).

cells (17). This finding suggests that NKAP functions as an oncogene in the proliferation and invasion of colon cancer cells. In addition, NKAP was found to regulate the EMT by modulating the expression of $\beta$-catenin and E-cad in colon cancer cells. $\beta$-catenin has been reported to accumulate in the invasive front of colon cancer $(18,19)$. E-cad, a key epithelial marker protein, is involved in metastasis, drug resistance and prognosis of colon cancer (20-22). Therefore, the regulation of NKAP on EMT may be involved in its effect on cell invasion.

Apoptosis is a major regulatory mechanism for the control of cell growth, while dysregulation of apoptosis and resistance to cell death are common hallmarks of cancer (23). Promotion of apoptosis is an important aspect of cancer therapy $(24,25)$, Additionally, loss of NKAP is reported to promote apoptosis in HSCs cells (9). Herein, our results showed that NKAP knockdown significantly increased the rate of apoptosis in colon cancer cells in vitro, while NKAP overexpression inhibited apoptosis. The intrinsic mitochondrial pathway is a pivotal mechanism for the regulation of apoptosis (26). However, it is reported that tumor cells generally evade regulation of apoptosis by impairing the expression of anti-apoptotic or pro-apoptotic proteins (27). It is widely held that the Caspase and Bcl-2 families are key apoptosis-related proteins involved in initiating the mitochondrial apoptosis pathway (28-30). As described above, we found that loss of NKAP decreased the expression of anti-apoptotic protein $\mathrm{Bcl}-2$ and increased the expression of pro-apoptotic proteins Bax and Active Caspase-3, while NKAP overexpression caused the opposite effect (Fig. 4). These findings suggest that NKAP knockdown could trigger cell apoptosis in a mitochondrial pathway in colon cancer in vitro. In addition, we detected the effect of
NKAP on autophagy in colon cancer cells. Autophagy is a complex cellular process regulated by multiple signaling pathways (31). Autophagy has been reported to play different roles in different tumor model systems, suppressing or promoting cancer cell proliferation or tumorigenesis, indicating that the effect of autophagy on cancer progression is context-dependent $(32,33)$. During autophagy, the cytosolic form of LC3, LC3-I, is translocated to the autophagic membrane-bound form, LC3-II. Therefore, the relative expressions of LC3-I and II are considered to be an index of autophagy $(34,35)$. In the present study, loss of NKAP resulted in decreased LC3-I level and increased LC3-II level in colon cancer cells, and NKAP overexpression causes the opposite effect, indicating that NKAP could regulate cell autophagy in colon cancer cells, and silencing NKAP promotes autophagy.

NKAP has been shown to function as an RNA-binding protein in RNA splicing (11). Li et al have confirmed that NKAP functions as a mitotic regulator in chromosome alignment, and loss of NKAP results in misalignment of chromosomes which leads to mitotic arrest in human soft tissue sarcomas (12). However, the specific mechanism of NKAP in colon cancer remains unclear. The PI3K/Akt signaling pathway has been implicated in various cellular processes and is reported to play an important role in the initiation and progression of colon cancer by regulating a series of oncogenes or anti-oncogenes $(36,37)$. Our data revealed that the Akt/mTOR pathway was overactivated in colon cancer through upregulation of p-Akt and p-mTOR (Fig. S1B and D). Herein, we found that NKAP knockdown significantly inhibited activation of the Akt/mTOR pathway, which was further confirmed by decreased expression of downstream 
proteins, Cyclin D1 and P70 (Fig. 4A). Cyclin D1 is a pivotal proto-oncogene involved in cancer progression by cell cycle regulation (38). P70 is a serine/threonine protein kinase that has been confirmed to be positively associated with tumor growth and angiogenesis (39). Additionally, we found that the $\mathrm{NF}-\kappa \mathrm{B}$ pathway was also suppressed as NKAP was knocked down and upregulated in NKAP overexpression cells. Based on the above results, we are able to conclude that the Akt $/ \mathrm{mTOR}$ and NF- $\kappa \mathrm{B}$ pathways may be involved in the oncogenic role of NKAP in colon cancer.

In summary, to the best of our knowledge, results of the present study reveal for the first time that NKAP is upregulated in colon cancer and plays an oncogenic role in the progression of colon cancer, whereby NKAP knockdown suppresses the proliferation and invasion of colon cancer cells, as well as promote apoptosis and autophagy. Therefore, our study provides new insight regarding the role of NKAP in tumor progression, which may provide a potential target for the treatment of colon cancer.

\section{Acknowledgements}

Not applicable.

\section{Funding}

This study was supported by National Natural Science Foundation of China (nos. 81502508 and 81600121) and Natural Science Foundations of Shandong Province (no. ZR2016HQ46).

\section{Availability of data and materials}

The datasets used and/or analyzed during the current study are available from the corresponding author on reasonable request.

\section{Authors' contributions}

WS, GL, JH and XL participated in the design of the study and drafted the manuscript. YD, AF and ZC participated in data analyses and helped draft the manuscript. All authors gave final approval for publication.

\section{Ethics approval and consent to participate}

Primary human tumor specimen collection was granted prior approval from the Ethics Committee of Shandong Provincial Hospital Affiliated to Shandong University (no. 2018-220), Jinan, China. Tissue analysis was approved by the Ethics Committee of Shandong Provincial Hospital Affiliated to Shandong University. All of the patients provided informed consent to participate.

\section{Patient consent for publication}

Not applicable.

\section{Competing interests}

The authors declare that they have no competing interests.

\section{References}

1. Torre LA, Bray F, Siegel RL, Ferlay J, Lortet-Tieulent J and Jemal A: Global cancer statistics, 2012. CA Cancer J Clin 65: 87-108, 2015.

2. Siegel RL, Miller KD, Fedewa SA, Ahnen DJ, Meester RGS, Barzi A and Jemal A: Colorectal cancer statistics, 2017. CA Cancer J Clin 67: 177-193, 2017.

3. Vatandoust S, Price TJ and Karapetis CS: Colorectal cancer: Metastases to a single organ. World J.Gastroenterol 21: 11767-11776, 2015.

4. Siegel R, Ma J, Zou Z and Jemal A: Cancer statistics, 2014. CA Cancer J Clin 64: 9-29, 2014.

5. Chen D, Li Z, Yang Q, Zhang J, Zhai Z and Shu HB: Identification of a nuclear protein that promotes NF-kappaB activation. Biochem Biophys Res Commun 310: 720-724, 2003.

6. Pajerowski AG, Nguyen C, Aghajanian H, Shapiro MJ and Shapiro VS: NKAP is a transcriptional repressor of notch signaling and is required for T cell development. Immunity 30 : 696-707, 2009.

7. Thapa P, Das J, Mcwilliams D, Shapiro M, Sundsbak R, Nelson-Holte M, Tangen S, Anderson J, Desiderio S, Hiebert $\mathrm{S}$, et al: The transcriptional repressor NKAP is required for the development of iNKT cells. Nat Commun 4: 1582, 2013.

8. Hsu FC, Pajerowski AG, Nelson-Holte M, Sundsbak R and Shapiro VS: NKAP is required for T cell maturation and acquisition of functional competency. J Exp Med 208: 1291-1304, 2011.

9. Pajerowski AG, Shapiro MJ, Gwin K, Sundsbak R, Nelson-Holte M, Medina K and Shapiro VS: Adult hematopoietic stem cells require NKAP for maintenance and survival. Blood 116: 2684-2693, 2010.

10. Thapa P, Chen MW, Mcwilliams DC, Belmonte P, Constans M, Sant'Angelo DB and Shapiro VS: NKAP regulates invariant NKT cell proliferation and differentiation into ROR- $\gamma \mathrm{t}$-expressing NKT17 cells. J Immunol 196: 4987-4998, 2016.

11. Burgute BD, Peche VS, Steckelberg AL, Glöckner G, Gaßen B, Gehring NH and Noegel AA: NKAP is a novel RS-related protein that interacts with RNA and RNA binding proteins. Nucleic Acids Res 42: 3177-3193, 2014.

12. Li T, Chen L, Cheng J, Dai J, Huang Y, Zhang J, Liu Z, Li A, Li N, Wang H, et al: SUMOylated NKAP is essential for chromosome alignment by anchoring CENP-E to kinetochores. Nat Commun 7: 12969, 2016.

13. Wu QB and Sun GP: Expression of COX-2 and HER-2 in colorectal cancer and their correlation. World J Gastroenterol 21: 6206-6214, 2015.

14. Livak KJ and Schmittgen TD: Analysis of relative gene expression data using real-time quantitative PCR and the 2(-Delta Delta C(T)) method. Methods 25: 402-408, 2001.

15. Cotter TG: Apoptosis and cancer: The genesis of a research field. Nat Rev Cancer 9: 501-507, 2009.

16. Tang X, Zha L, Li H, Liao G, Huang Z, Peng X and Wang Z: Upregulation of GNL3 expression promotes colon cancer cell proliferation, migration, invasion and epithelial-mesenchymal transition via the Wnt $/ \beta$-catenin signaling pathway. Oncol Rep 38: 2023-2032, 2017.

17. Liu J, Wang H, Yin Y, Li Q and Zhang M: NKAP functions as an oncogene and its expression is induced by $\mathrm{CoCl}_{2}$ treatment in breast cancer via AKT/mTOR signaling pathway. Cancer Manag Res 10: 5091-5100, 2018.

18. Oguma K, Oshima H, Aoki M, Uchio R, Naka K, Nakamura S, Hirao A, Saya H, Taketo MM and Oshima M: Activated macrophages promote Wnt signalling through tumour necrosis factor-alpha in gastric tumour cells. EMBO J 27: 1671-1681, 2008.

19. Amado NG, Predes D, Moreno MM, Carvalho IO, Mendes FA and Abreu JG: Flavonoids and Wnt/ $\beta$-catenin signaling: Potential role in colorectal cancer therapies. Int J Mol Sci 15: 12094-12106, 2014.

20. Saito T, Masuda N, Miyazaki T, Kanoh K, Suzuki H, Shimura T, Asao T and Kuwano H: Expression of EphA2 and E-cadherin in colorectal cancer: Correlation with cancer metastasis. Oncol Rep 11: 605-611, 2004.

21. Murray L: The role of E-cadherin in colon cancer drug resistance. Univ Glasgow, 2010.

22. Kwak JM, Min BW, Lee JH, Choi JS, Lee SI, Park SS, Kim J, Um JW, Kim SH and Moon HY: The prognostic significance of E-cadherin and liver intestine-cadherin expression in colorectal cancer. Dis Colon Rectum 50: 1873-1880, 2007. 
23. Croce CM and Reed JC: Finally, an apoptosis-targeting therapeutic for cancer. Cancer Res 76: 5914-5920, 2016.

24. Fesik SW: Promoting apoptosis as a strategy for cancer drug discovery. Nat Rev Cancer 5: 876-885, 2005.

25. Huang Q, Li S, Cheng P, Deng M, He X, Wang Z, Yang CH, Zhao XY and Huang J: High expression of anti-apoptotic protein $\mathrm{Bcl}-2$ is a good prognostic factor in colorectal cancer: Result of a meta-analysis. World J Gastroenterol 23: 5018-5033, 2017.

26. Parrish AB, Freel CD and Kornbluth S: Cellular mechanisms controlling caspase activation and function. Cold Spring Harb Perspect Biol 5, 2013.

27. Igney FH and Krammer PH: Death and anti-death: Tumour resistance to apoptosis. Nat Rev Cancer 2: 277-288, 2002.

28. Gui D, Guo Y, Wang F, Liu W, Chen J, Chen Y, Huang J and Wang N: Astragaloside IV, a novel antioxidant, prevents glucose-induced podocyte apoptosis in vitro and in vivo. PLoS One 7: e39824, 2012.

29. Martinou JC and Youle RJ: Mitochondria in apoptosis: Bcl-2 family members and mitochondrial dynamics. Dev Cell 21 : 92-101, 2011.

30. Wu R, Tang S, Wang $\mathrm{M}, \mathrm{Xu} \mathrm{X}$, Yao $\mathrm{C}$ and Wang S: MicroRNA-497 induces apoptosis and suppresses proliferation via the Bcl-2/Bax-caspase9-caspase3 pathway and cyclin D2 protein in HUVECs. PLoS One 11: e0167052, 2016.
31. He C and Klionsky DJ: Regulation mechanisms and signaling pathways of autophagy. Annu Rev Genet 43: 67-93, 2009.

32. White E: Deconvoluting the context-dependent role for autophagy in cancer. Nat Rev Cancer 12: 401-410, 2012.

33. White E: The role for autophagy in cancer. J Clin Invest 125 : 42-46, 2015.

34. Burman C and Ktistakis NT: Autophagosome formation in mammalian cells. Semin Immunopathol 32: 397-413, 2010.

35. Tan S, Shi H, Ba M, Lin S, Tang H, Zeng $X$ and Zhang $X$ miR-409-3p sensitizes colon cancer cells to oxaliplatin by inhibiting Beclin-1-mediated autophagy. Int J Mol Med 37: 1030-1038, 2016.

36. Jiang QG, Li TY, Liu DN and Zhang HT: PI3K/Akt pathway involving into apoptosis and invasion in human colon cancer cells LoVo. Mol Biol Rep 41: 3359-3367, 2014.

37. Malinowsky K, Nitsche U, Janssen KP, Bader FG, Späth C, Drecoll E, Keller G, Höfler H, Slotta-Huspenina J and Becker KF: Activation of the PI3K/AKT pathway correlates with prognosis in stage II colon cancer. Br J Cancer 110: 2081-2089, 2014.

38. Alao JP: The regulation of Cyclin D1 degradation: Roles in cancer development and the potential for therapeutic invention. Mol Cancer 6: 24, 2007.

39. Jiang BH: PI3K/AKT and mTOR/p70S6K1 signaling pathways in human cancer. Curr Cancer Drug Targets 13: 233, 2013. 